Journal of Computer Science 7 (12): 1900-1907, 2011

ISSN 1549-3636

(C) 2011 Science Publications

\title{
An Improved Face Recognition Technique Based on Modular LPCA Approach
}

\author{
${ }^{1}$ Mathu Soothana S. Kumar, ${ }^{1}$ Retna Swami and ${ }^{2}$ Muneeswaran Karuppiah \\ ${ }^{1}$ Department of Information Technology, \\ Noorul Islam College of Engineering, Kumaracoil, Thuckalay, \\ ${ }^{2}$ Department of Computer Science and Engineering, \\ Mepco Schlenk Engineering College, Sivakasi, India
}

\begin{abstract}
Problem statement: A face identification algorithm based on modular localized variation by Eigen Subspace technique, also called modular localized principal component analysis, is presented in this study. Approach: The face imagery was partitioned into smaller sub-divisions from a predefined neighborhood and they were ultimately fused to acquire many sets of features. Since a few of the normal facial features of an individual do not differ even when the pose and illumination may differ, the proposed method manages these variations. Results: The proposed feature selection module has significantly, enhanced the identification precision using standard face databases when compared to conservative and modular PCA techniques. Conclusion: The proposed algorithm, when related with conservative PCA algorithm and modular PCA, has enhanced recognition accuracy for face imagery with illumination, expression and pose variations.
\end{abstract}

Key words: Face recognition, feature extraction, Pose invariance, illumination invariance, feature vector, partial occlusion, precise class, recognition accuracy

\section{INTRODUCTION}

The face plays a major role towards social interaction for identity and emotion. Recognizing faces (Zhao et al., 2003) through computers can be applied to a broad category of problems including security systems, image and film processing, criminal identification and human-computer interaction. Criminal identification can be greatly improved by modeling a face, which may differ from a large set of face models in a stored database. Several methods have been focused over the last decade, in which lowdimensional representations (Sirovitch and Kirby, 1987) of the image are used to carry out identification. These methods, often termed as appearance-based methods (Cootes et al., 2001), are different from feature-based techniques in that low-dimensional representation is, in least-squares logic, close to the original image. Here, the feature vectors are used for classification in a linear projection of the face image into a lower-dimensional linear subspace. In extreme cases, the feature vector is chosen as a whole image, with every element in the feature vector taken from a pixel in the image.
The main focus of earlier research was to improve the accuracy of face recognition subjected to varying facial expression (Zhang and Martinez, 2004) illumination (Adini et al., 1997) and head pose (Georghiades et al., 2001). Among them, PCA and modular PCA (MPCA) are the popular techniques in facial image recognition. Conservative PCA technique is not highly accurate, when the illumination and pose of the facial images vary considerably. The MPCA method (Pentland et al., 1994; Gottumukkal and Asari, 2004) is an extension of the conservative PCA in which, the face images are divided into smaller images and the PCA method is applied on each of them, for better performance under the conditions of large variations in illumination and expression. But this did not yield better results for large pose variations. Though, the whole face image may be affected due to variations such as pose, illumination, expression and partial occlusions (Martinez, 2002), facial variations are restricted mostly to local regions (Martinez, 2000; Ahmed, 2010; Zou et al., 2007). Hence in this research study, an attempt is made to improve the accuracy of recognition under the conditions of varying facial expression, illumination and pose by using modular localized variation

Corresponding Author: Mathu Soothana S. Kumar, Department of Information Technology,

Noorul Islam College of Engineering, Kumaracoil, Thuckalay, India Tell: +919486856119 
(Kumar and Jegadeesh, 2011) in Eigen subspace (LPCA). Here, the variations can be focused while modularizing the images, where the modules created are sufficiently small. The main drawback of large number of modules is that it eliminates the dependencies that occur among various neighboring pixels. To overcome this problem, a module creation tactic is introduced which considers additional pixel dependencies across various sub regions thereby classification accuracy can be improved.

Related works: PCA based methods contributes dimensionality reduction. The modularization of PCA called modular PCA is addressed.

Principal component analysis: PCA (Sirovitch and Kirby, 1987; Turk and Pentland, 1991a; Yuen et al., 2009) is a popular geometric approach for facial image identification, in which, face images are articulated as a subset of their Eigen vectors called Eigen faces. A technique that is used for dimensionality reduction in computer vision - predominantly in facial recognition is principal component analysis. A set of images corresponds to, a set of points in a high dimensional space. Since, facial images are similar in composition, these points will not be randomly distributed and therefore can be described by a lower dimensional subspace. PCA assigns the basis vector for this lower dimensional space called face space, also called the Eigen vector, which may be calculated from covariance matrix of the original facial images. Let $1_{1}, 1_{2}, \ldots$, $\mathrm{I}_{\mathrm{M}}$ be the training set of face images. The average face is defined by Eq. 1:

$$
\mathrm{A}=\frac{1}{\mathrm{M}} \sum_{\mathrm{i}=1}^{\mathrm{M}} \mathrm{I}_{\mathrm{i}}
$$

Each face differs from the average face by the vector. The covariance matrix $\mathrm{C}$ is obtained by Eq. 2 :

$\mathrm{C}=\frac{1}{\mathrm{M}} \sum_{\mathrm{i}=1}^{\mathrm{M}} \mathrm{Y}_{\mathrm{i}} \mathrm{Y}_{\mathrm{i}}^{\mathrm{T}}$

The Eigen vectors of the covariance matrix are computed and the E' significant Eigen vectors are chosen as those with the largest corresponding Eigen values. From these Eigen vectors, the weights for each image in the training set are computed by Eq. 3:

$$
\mathrm{W}_{\mathrm{IK}}=\mathrm{E}_{\mathrm{K}}^{\mathrm{T}}\left(\mathrm{I}_{\mathrm{i}}-\mathrm{A}\right) \forall \mathrm{i}, \mathrm{K}
$$

where, $E_{K}$ represents the Eigen vectors corresponding to the $\mathrm{E}$ ' largest Eigen values of $\mathrm{C}$ and $\mathrm{K}$ which vary from 1 to E'. PCA encodes the pattern information based on second order dependencies, i.e., pixel wise covariance among the pixels and are insensate to the dependencies of multiple (more than two) pixels in the patterns. The principal components are uncorrelated because the Eigen values that may be calculated in PCA (Turk and Pentland, 1991b) should satisfy ortho-normal property.

Modular principal component analysis: The PCA based face recognition technology is not much effective under the environment of varying pose and illumination, as this technology deems the global information of each face image and represents them with a set of weights. Under this environment, the weight vectors are varying significantly across normal and varying pose and illumination. Therefore it is not easy to recognize them correctly. At the same time if the face imagery is split into tiny regions and the weight vectors are calculated for each of these locations, then the weights will be more representative of the local information of the face is defined by (4). While there is a variation in the pose or illumination, only some of the face locations would vary and the rest of the regions would remain similar as the face regions of a normal image. For this reason, weights of the face areas not affected by varying pose and illumination will closely match with the weights of the same individual face areas under normal conditions. Hence, it is anticipated that enhanced identification rate can be acquired by the MPCA approach (Pentland et al., 1994; Gottumukkal and Asari, 2004). If the face imagery is split into tiny regions, the global information of the face may be lost and the precision of this method may depreciate Eq.4:

$$
\mathrm{I}_{\mathrm{ij}}(\mathrm{m}, \mathrm{n})=\mathrm{I}_{\mathrm{i}}\left(\frac{\mathrm{L}}{\sqrt{\mathrm{N}}}(\mathrm{j}-1)+\mathrm{m}, \frac{\mathrm{L}}{\sqrt{\mathrm{N}}}(\mathrm{j}-1)+\mathrm{n}\right) \forall \mathrm{i}, \mathrm{j}
$$

where, $\mathrm{i}$ varies from 1 to $\mathrm{M}, \mathrm{M}$ being the number of images in the training set, $\mathrm{j}$ varies from 1 to $\mathrm{N}, \mathrm{N}$ being the number of sub-images and $\mathrm{m}$ and $\mathrm{n}$ vary from 1 to $\mathrm{L} / \sqrt{\mathrm{N}}$ and $\mathrm{L} \times \mathrm{L}$ is the size of the image. When the image is partitioned, for each partition the PCA method is applied. The modular PCA method entirely outperforms the PCA method in all aspects for the image partitions at 4, 16 and 64. Nevertheless, better results were obtained when the image partition is of size 16 . 


\section{MATERIALS AND METHODS}

Here, we discuss the creation of many sub- images retaining the dependencies among the neighboring pixels. Local facial variation caused by expression, pose, illumination etc., may be dealt with more efficiently by providing a region-based feature extraction approach. This can enhance the categorization capability. Splitting the images into adequately smaller modules will help in localizing the facial variations. Figure 1 depicts the model of localization of variations and pixel dependence exposed because of face accessories. The localization of these variations gets improved with tiny modules, whereas a huge amount of dependencies among various nearby pixels are discarded. The number of modules to be traded is explained in the following paragraph.

Neighborhood defined modular spaces: The face imagery is normalized to a size $64 \times 64$ in our research to reduce the processing speed. In MPCA method, each face image is partitioned into several non-overlapping sub-images (Gottumukkal and Asari, 2004). We split the $64 \times 64$ face image into 16 numbers block of size $16 \times 16$. In addition, each block is partitioned into many $4 \times 8$ blocks which are fused to form as several $8 \times 8$ blocks. Twenty eight such different $8 \times 8$ pixel blocks can be formed within the neighborhood region of size $16 \times 16$. Fig. 2 elucidates the process of obtaining such blocks from each of the $16 \times 16$ pixels neighborhood. Therefore a total of 448 blocks can be acquired from a $64 \times 64$ face image and Eigen spaces are created for each such block.

A similar method of block creation is applied for the query image and each block is projected onto the respective Eigen space and classified based on minimum distance measure. A voting procedure concludes the result of the overall classification by considering the individual classification results of all the 448 blocks. Every block is classified into a precise class and the class which acquires the maximum number of blocks in its favor is considered the winner which is highlighted in Fig. 3.

Several experimental results have been presented with block size $16 \times 16$ on face images of size $16 \times 16$, $32 \times 32,64 \times 64,128 \times 128$ and $256 \times 256$. It has been observed that maximum recognition precision is obtained when the image of size $64 \times 64$ was partitioned into blocks of size $16 \times 16$. The algorithm (compute LPCAFV) splits the given image into as many numbers of blocks of size $16 \times 16$. Each block is split into many $4 \times 8$ sub blocks and merged to give $8 \times 8$.

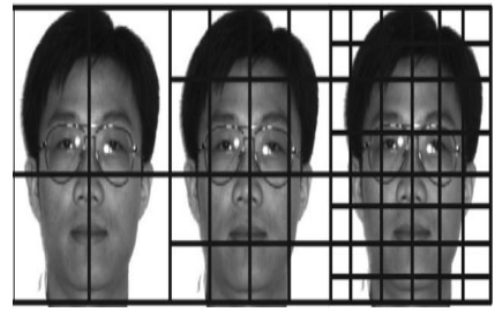

Fig. 1: Illustration of the concept of localization of variations and pixel dependencies for varying modules

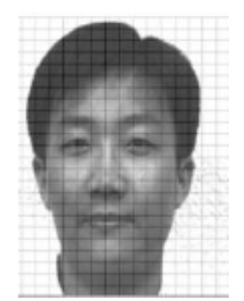

(a)

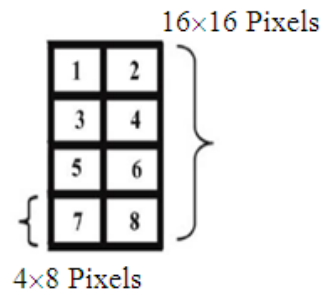

(b)

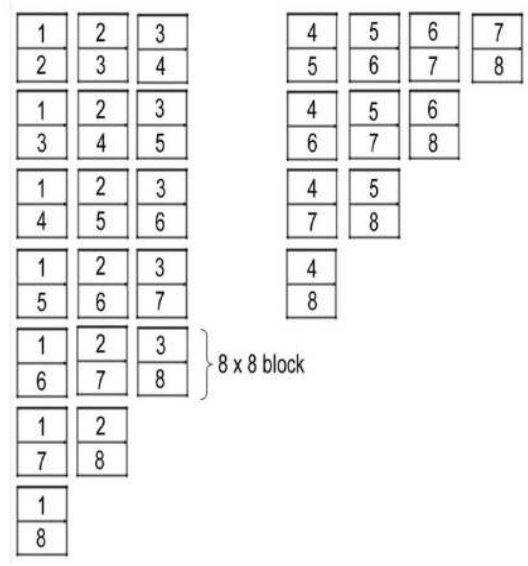

(c)

Fig. 2: Creation of blocks of size $8 \times 8$ by fusing blocks of $4 \times 8$ within $16 \times 16$ pixels (a) Original image (b) $16 \times 16$ blocks (c) Creation of $8 \times 8$ blocks 


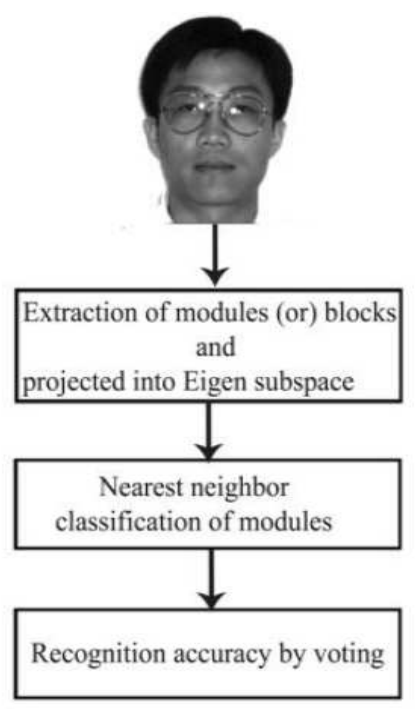

Fig. 3: Concept of classification of blocks (or) modules by voting

Algorithm compute LPCAFV $\left(\mathrm{I}_{\mathrm{T}}\right)$

\{

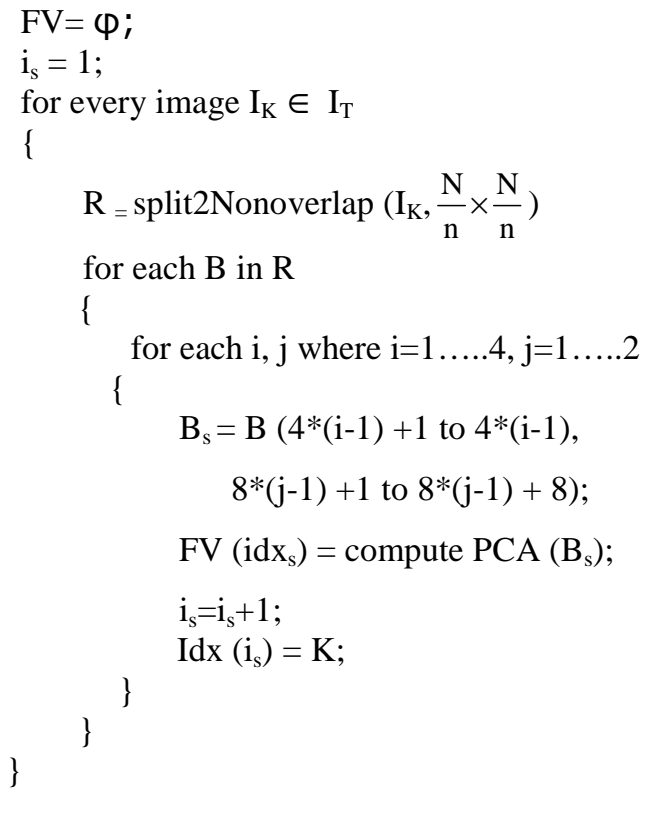

Sub-images on neighborhood basis to retain the localized features. The techniques given in Eq. 1-3 are used to compute the PCA of the sub-images that are formed of size $8 \times 8$. Apart from computing the PCA for each sub-image, the Eigen space for each sub-image has to be maintained which is its overhead of the proposed method.

\section{RESULTS AND DISCUSSION}

The performance of the PCA, MPCA and LPCA was evaluated with three image databases, UMIST, ORL and Yale. The UMIST database consists of images with varying pose; the ORL database consists of images with varying expression, lightning and details; and the Yale database consists of images with varying illumination and expression. Table 1 shows the details of experimental image databases.

We split the total number of images into training and testing which are disjoint. The number of features in each block is 64. Using LPCA method, the significant features ranging from 1-64 are considered and the classification rates are noted. Also, the numbers of training images were varied from $30-90 \%$ and the remaining images were used for testing and the classification rates are noted. Tables 2 and 3 show the recognition accuracy of PCA, MPCA and LPCA using varying number of Eigen vectors.

From the performance of all the three algorithms, it is noticed that the proposed method yields better recognition accuracy as shown in Fig. 4. It is observed that MPCA shows better performance than PCA. Also, we observe from the results that the proposed method outperforms the PCA and MPCA methods in all aspects with significant improvement under the condition of varying pose. However, best results were obtained for an image of size $64 \times 64$ with block size $16 \times 16$.

Results for pose variation: Here, we conducted tests for large pose variations using the images in the UMIST database for the three methods to calculate the recognition accuracy (or) True Positive and False Rejection Rate (FRR). In LPCA, initially the block size $16 \times 16$ is considered, thereafter splitting into $4 \times 8$ sub blocks is performed and then fusing them leading to 28 blocks. Instead of considering images of fixed size of $64 \times$, different image sizes were considered such as $16 \times 16,32 \times 32,64 \times 64,128 \times 128,256 \times 256$ and experimented. Figure 5 shows the recognition accuracy and FRR for the LPCA method with varying size of an image. In (Gottumukkal and Asari, 2004), several results have been highlighted for $80 \%$ training in PCA and MPCA. In our work, we achieved better results for $50 \%$ training and the results for $80 \%$ training has also been highlighted. In the case of PCA, the recognition accuracy was 30\% and FRR was $70 \%$ and in MPCA, the recognition accuracy was $34 \%$ and FRR was $66 \%$. The results obtained by LPCA for UMIST are shown in Fig. 5. 


\begin{tabular}{llll}
\multicolumn{4}{l}{ Table 1: Experimental image databases } \\
\hline & $\begin{array}{l}\text { Number } \\
\text { of classes }\end{array}$ & $\begin{array}{l}\text { Number } \\
\text { of samples } \\
\text { in each class }\end{array}$ & Image \\
Database size & 10 & 20 & $112 \times 92$ \\
\hline UMIST & 40 & 10 & $112 \times 92$ \\
ORL & 15 & 11 & $243 \times 320$ \\
YALE & 15 & 10 & $243 \times 320$ \\
YALE ONE image left & 15 &
\end{tabular}

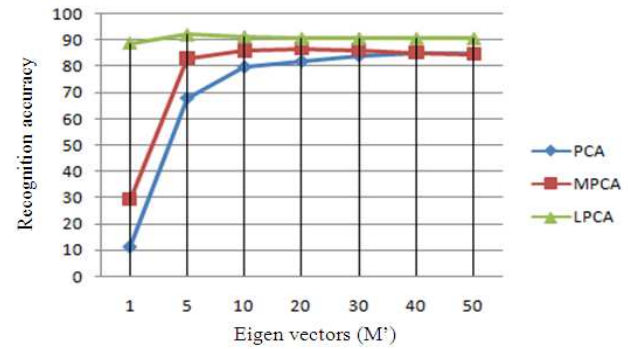

(a)

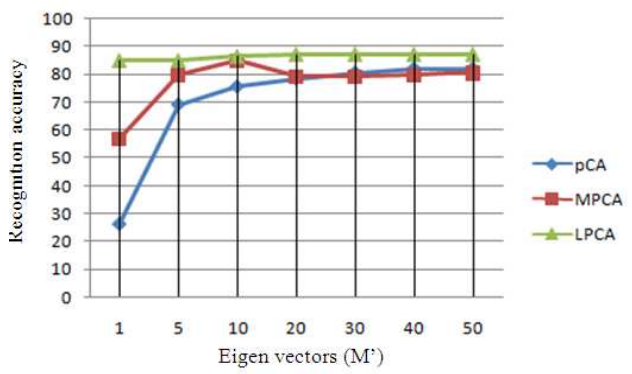

(b)

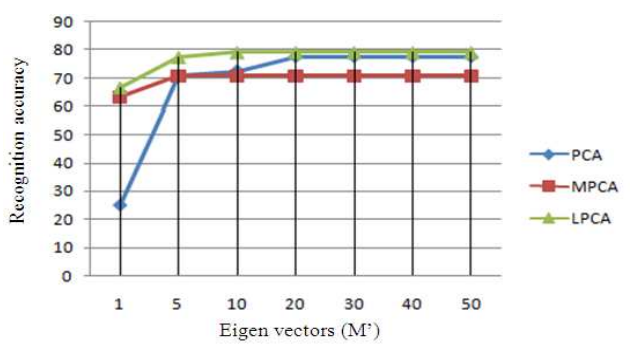

(c)

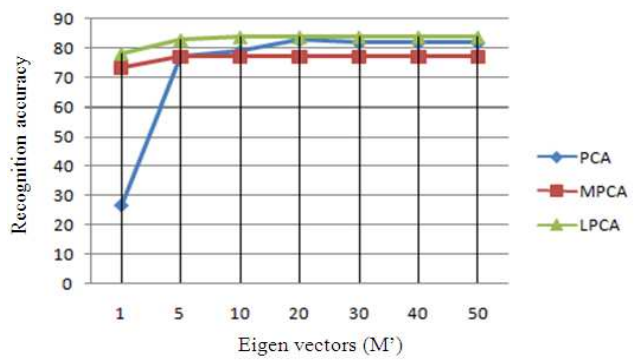

(d)

Fig. 4: Recognition accuracy of LPCA with varying Eigen vectors (M') for $30 \%$ training. (a) ORL database 30\% training. (b) UMIST database 30\% training. (c) YALE database $30 \%$ training. (d)YALE database one image left $30 \%$ training

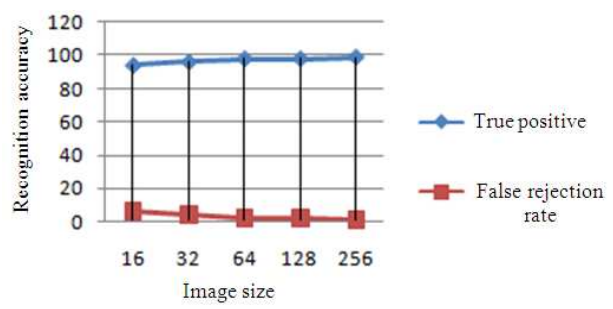

(a)

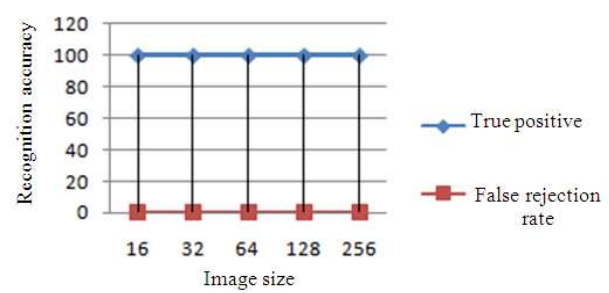

(b)

Fig. 5: Recognition accuracy and FRR for the LPCA method with varying size of an image (UMIST) (a) $50 \%$ training (b) $80 \%$ training

In LPCA the recognition accuracy was $98 \%$ and FRR was 2 for $50 \%$ training and recognition accuracy of 100 and FRR of 0 for $80 \%$ training for an image size of $64 \times 64$ with block size 16 . Hence, LPCA has substantial improvement over the PCA and MPCA under the condition of varying pose.

Results for expression and illumination variation: Here, we conducted tests for large expression and illumination variations using the images in the ORL and Yale databases to calculate the recognition accuracy and FRR. As before, we vary the size of an image from $16 \times 16,32 \times 32, \quad 64 \times 64,128 \times 128$ and $256 \times 256$ to observe the effect it has on face recognition. In our work, we achieved better results for 50\% training and the result for $80 \%$ training has also been highlighted. Figure 6 shows the recognition accuracy and FRR for the LPCA method for ORL and for YALE in Fig. 7 with varying image. In the case of PCA, the recognition accuracy was $44 \%$ and FRR was $56 \%$. In MPCA, the recognition accuracy was 84 and FRR was $16 \%$. In LPCA, the recognition accuracy was $97.5 \%$ and FRR was $2.5 \%$ for $50 \%$ training and recognition accuracy of $100 \%$ and FRR of $0 \%$ for $80 \%$ training for ORL database. Similarly, for LPCA, the recognition accuracy was $86.67 \%$ and FRR was $13.33 \%$ for $50 \%$ training and recognition accuracy of $93.3 \%$ and FRR of $6.7 \%$ for $80 \%$ training for YALE database. 
J. Computer Sci., 7 (12): 1900-1907, 2011

Table 2: Comparison of the highest accuracy achieved by several methods for 30\% training

\begin{tabular}{lcccccccc}
\hline Database & Method & $\mathrm{M}^{\prime}=1$ & $\mathrm{M}^{\prime}=5$ & $\mathrm{M}^{\prime}=10$ & $\mathrm{M}^{\prime}=20$ & $\mathrm{M}^{\prime}=30$ & $\mathrm{M}^{\prime}=40$ & $\mathrm{M}^{\prime}=50$ \\
\hline UMIST & PCA & 26.4 & 69.3 & 75.7 & 78.6 & 80.7 & 82.1 & 82.1 \\
& MCPA & 57.1 & 80.0 & 85.0 & 79.2 & 79.3 & 80.0 & 80.7 \\
\multirow{3}{*}{ ORL } & LPCA & 85.0 & 85.0 & 86.4 & 87.1 & 87.1 & 87.1 & 87.1 \\
& PCA & 11.4 & 67.9 & 79.6 & 81.8 & 83.9 & 85.0 & 85.0 \\
& MCPA & 29.3 & 83.2 & 86.1 & 86.8 & 86.4 & 85.4 & 84.6 \\
YALE & LPCA & 88.9 & 92.1 & 91.4 & 90.7 & 90.7 & 90.7 & 90.7 \\
& PCA & 25.0 & 70.8 & 72.5 & 77.5 & 77.5 & 77.5 & 77.5 \\
\multirow{3}{*}{ YALE ONE image left } & MCPA & 63.3 & 70.8 & 70.8 & 70.8 & 70.8 & 70.8 & 70.8 \\
& LPCA & 66.7 & 77.5 & 79.1 & 79.1 & 79.2 & 79.2 & 79.2 \\
& PCA & 26.7 & 77.1 & 79.0 & 82.9 & 81.9 & 81.9 & 81.9 \\
& MCPA & 73.3 & 77.1 & 77.1 & 77.1 & 77.1 & 77.1 & 77.1 \\
& LPCA & 78.1 & 82.9 & 83.8 & 83.8 & 83.8 & 83.8 & 83.8 \\
\hline
\end{tabular}

Table 3: Comparison of the highest accuracy achieved by several methods for $90 \%$ training

\begin{tabular}{llrrrrrrr}
\hline Database & Method & $\mathrm{M}^{\prime}=1$ & \multicolumn{1}{c}{$\mathrm{M}^{\prime}=5$} & $\mathrm{M}^{\prime}=10$ & $\mathrm{M}^{\prime}=20$ & $\mathrm{M}^{\prime}=30$ & $\mathrm{M}^{\prime}=40$ & $\mathrm{M}^{\prime}=50$ \\
\hline UMIST & PCA & 55.0 & 95.0 & 95.0 & 100.0 & 100.0 & 100.0 & 100.0 \\
& MCPA & 65.0 & 100.0 & 100.0 & 100.0 & 100.0 & 100.0 & 100.0 \\
\multirow{5}{*}{ ORL } & LPCA & 100.0 & 100.0 & 100.0 & 100.0 & 100.0 & 100.0 & 100.0 \\
& PCA & 35.0 & 85.0 & 95.0 & 97.5 & 97.5 & 97.5 & 97.2 \\
\multirow{5}{*}{ YALE } & MCPA & 47.5 & 90.0 & 95.0 & 100.0 & 100.0 & 100.0 & 100.0 \\
& LPCA & 92.5 & 97.5 & 97.5 & 100.0 & 100.0 & 100.0 & 100.0 \\
& PCA & 23.3 & 80.0 & 90.0 & 90.0 & 93.3 & 93.3 & 93.3 \\
YALE ONE image left & MCPA & 53.3 & 86.7 & 90.0 & 93.3 & 93.3 & 93.3 & 93.3 \\
& LPCA & 60.0 & 90.0 & 90.0 & 93.3 & 93.3 & 93.3 & 93.3 \\
& PCA & 46.7 & 93.3 & 93.3 & 93.3 & 93.3 & 93.3 & 93.3 \\
& MCPA & 60.0 & 93.3 & 93.3 & 93.3 & 93.3 & 93.3 & 93.3 \\
& LPCA & 66.7 & 93.3 & 93.3 & 93.3 & 93.3 & 93.3 & 93.3 \\
\hline
\end{tabular}

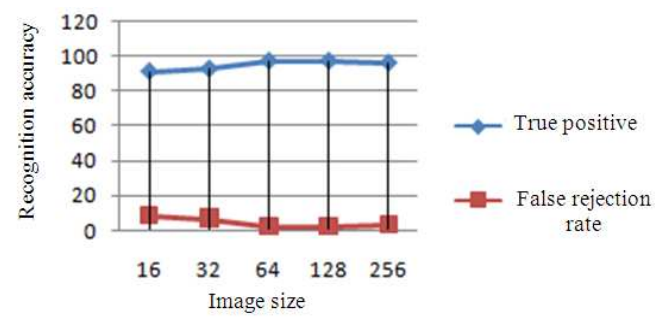

(a)

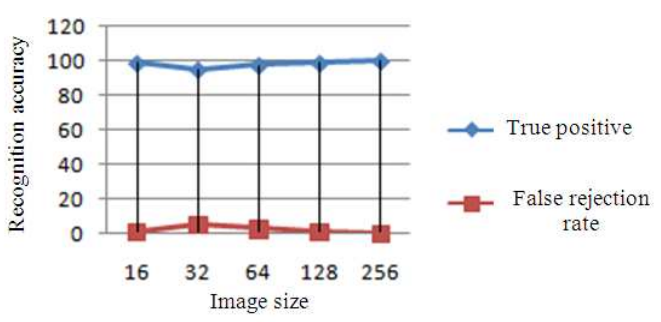

(b)

Fig. 6: Recognition accuracy and FRR for the LPCA method with varying size of an image (ORL) (a) $50 \%$ training (b) $80 \%$ training

A second set of experiments were performed by leaving out one image for testing. The results obtained for LPCA for Yale are shown in Fig. 8. For PCA, recognition accuracy was $48 \%$ and FRR was $52 \%$.

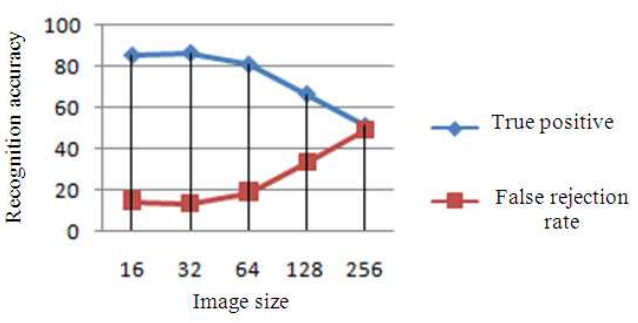

(a)

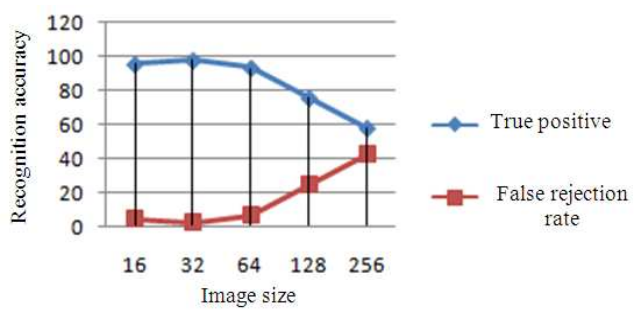

(b)

Fig. 7: Recognition accuracy and FRR for the LPCA method with varying size of an image (YALE) (a) $50 \%$ training (b) $80 \%$ training

In MPCA, the recognition accuracy was $78 \%$ and FRR was $22 \%$. In LPCA, the recognition accuracy was $94.67 \%$ and FRR was 5.33 for 50\% training and recognition accuracy of 96.67 and FRR of 3.33 for $80 \%$ training. 


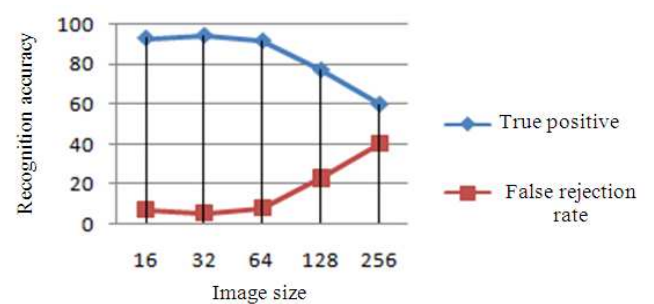

(a)

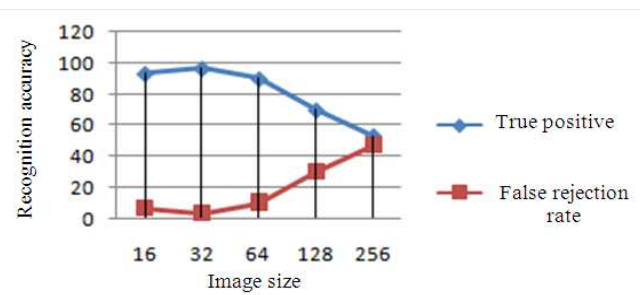

(b)

Fig. 8: Recognition accuracy and FRR for the LPCA method with varying size of an image (YALE with one Image left) (a) $50 \%$ training (b) $80 \%$ training

We can observe from the results that the LPCA method completely outperforms the PCA and MPCA method in all aspects, specifically for the image size of $16 \times 16,32 \times 32$ and $64 \times 64$ with block size 16 .

\section{CONCLUSION}

This study highlights a face recognition technique using UMIST, ORL and YALE face images. The feature selection strategy is robust to pose, illumination, expression and partial occlusions in the face images. A modular localized variation using Eigen space approach, also called LPCA for face recognition, has been proposed. For large variations in pose, illumination, expression and partial occlusions, the proposed LPCA method outperforms PCA and MPCA. The number of features is varied from 1-50 as against the total number of features of 64 and it is observed that 20 features are sufficient for reasonable recognition. The recognition accuracy is reasonable even at using $30 \%$ training set and improves very well at 50\% training set as against the existing methods. But all this happens at the cost of additional overhead of processing each sub-image. However, today computational resources could be exploited for fast response.

\section{REFERENCES}

Adini, Y., Y. Moses and S. Ullman, 1997. Face recognition: The problem of compensating for changes in illumination direction. IEEE Trans. Pattern Anal. Machine Intelli., 19: 721-732. DOI: 10.1109/34.598229

http://citeseer.ist.psu.edu/viewdoc/summary?doi=1 0.1.1.51.3607

Ahmed, I.A., 2010. Myocardial-infraction based on intelligent techniques. Am. J. Applied Sci., 7: 349351. DOI: 10.3844/ajassp.2010.349.351

Cootes, T.F., G.J. Edwards, C.J. Taylor, 2001. Active appearance models. IEEE Trans. Pattern Anal. Machine Intelli., 23: 681-685. DOI: 10.1109/34.927467

Georghiades, A.S., P.N. Belhumeur, D.J. Kriegman, 2001. From few too many: Illumination cone models for face recognition under variable lighting and pose. IEEE Trans. Pattern Anal. Machine Intelli., 23: 643-660. DOI: 10.1109/34.927464

Gottumukkal, R. and K.V. Asari, 2004. An improved face recognition technique based on modular PCA approach. Pattern Recognition Lett., 25: 429-436. DOI:10.1016/j.patrec.2003.11.005

Kumar, M.S.S.R. and J.S. Jegadeesh, 2011. A kernel modular Eigen space approach for facial recognition. International Conference on Intelligent Science and Technology (SUN IIST), India, pp: 1-9.

Martinez, A., 2002. Recognizing imprecisely localized, partially occluded and expression variant faces from a single sample per class. IEEE Trans. Pattern Analy. Machine Intelli., 24: 748-763, DOI:10.1109/TPAMI.2002.1008382

Martinez, A.M., 2000. Recognition of partially occluded and/or imprecisely localized faces using a probabilistic approach. IEEE Conf. Comput. Vision Pattern Recognition, 1: 712-717, DOI:10.1109/CVPR.2000.855890

Pentland, A., B. Moghaddam, T. Starner, 1994. Viewbased and modular eigen spaces for face recognition. IEEE Conference on Computer Vision and Pattern Recognition, 21-23 Jun, ieee Xploor, Seattle, WA, USA pp: 84-91, DOI: 10.1109/CVPR.1994.323814

Sirovitch, L. and M. Kirby, 1987. Low-dimensional procedure for the characterization of human faces. J. Optical Soc. Am., 4: 519-524, DOI:10.1364/JOSAA.4.000519

Turk, M. and A. Pentland, 1991a. Eigen faces for recognition. J. Cognitive Neuroscience, 3: 71-86. DOI: 10.1162/jocn.1991.3.1.71 
Turk, M. and A. Pentland, 1991b. Face recognition using eigen faces. IEEE Conf. on Computer Vision and Pattern Recognition, pp: 586-591, DOI: 10.1109/CVPR.1991.139758

Yuen, C.T., M. Rizon, W.S. San and T.C. Seong, 2009. Facial features for template matching based face recognition. Am. J. Applied Sci., 6: 1897. DOI: 10.3844/ajassp.2009.1897.1901

Zhang, Y. and A. Martinez, 2004. Recognition of expression variant faces using weighted subspaces. Proc. 17th Int. Conf. Pattern Recognition, ICPR, 3: 1051-4651. DOI: 10.1109/ICPR.2004.1334490
Zhao, W., R. Chellappa and A. Rosenfeld, 2003. Face recognition: A literature survey. ACM Comput. Sur., 35: 399-458.DOI:10.1145/954339.954342

Zou, J., Q. Ji and G. Nagy, 2007. A comparative study of local matching approach for face recognition, IEEE Trans. Imag. Proc., 16: 2617-2628. DOI: 10.1109/TIP.2007.904421 